\title{
Guillain-Barré syndrome in low-income and middle-income countries: challenges and prospects
}

\author{
Nowshin Papri ${ }^{1,2,7}$, Zhahirul Islam $\mathbb{1}^{1,7 凶}$, Sonja E. Leonhard $\mathbb{B}^{2}$, Quazi D. Mohammad ${ }^{3}$, \\ Hubert P. Endtz ${ }^{4,5}$ and Bart C. Jacobs (10 ${ }^{2,6}$
}

Abstract | The epidemiology, clinical characteristics, management and outcome of Guillain-Barré syndrome (GBS) differ between low-income and middle-income countries (LMIC) and highincome countries $(\mathrm{HIC})$. At present, limited data are available on GBS in LMIC and the true incidence of GBS in many LMIC remains unknown. Increased understanding of GBS in LMIC is needed because poor hygiene and high exposure to infections render populations in LMIC vulnerable to GBS outbreaks. Furthermore, insufficient diagnostic and health-care facilities in LMIC contribute to delayed diagnosis in patients with severe presentations of GBS. In addition, the lack of national clinical guidelines and absence of affordable, effective treatments contribute to worse outcomes and higher mortality in LMIC than HIC. Systematic population-based surveillance studies, cohort and case-control studies are required to understand the incidence and risk factors for GBS. Novel, targeted and cost-effective treatment strategies need to be developed in the context of health system challenges in LMIC. To ensure integrative rehabilitation services in LMIC, existing prognostic models must be validated, and responsive outcome measures that are cross-culturally applicable must be developed. Therefore, fundamental and applied research to improve the clinical management of GBS in LMIC should become a critical focus of future research programmes.

Guillain-Barré syndrome (GBS) is an immune-mediated polyradiculoneuropathy with an acute onset that affects 100,000 people worldwide annually ${ }^{1-3}$. GBS is characterized by rapidly progressive ascending weakness that initially affects the limbs and can eventually affect the cranial and respiratory muscles. Several infectious agents have been identified as triggers for the development of GBS, and clusters of this disease can be associated with outbreaks such as the Zika virus epidemic ${ }^{4-6}$. The severity of GBS is highly variable, ranging from mild distal limb weakness to complete paralysis, respiratory failure and even death. Several variants of GBS have been defined on the basis of their clinical presentation, including a pure motor variant, paraparetic variants and Miller Fisher syndrome (MFS) ${ }^{7,8}$, which is characterized by the clinical triad of ophthalmoplegia, ataxia and areflexia ${ }^{7}$. Several subtypes of GBS have also been identified on the basis of electrophysiological features ${ }^{1-3}$, including acute inflammatory demyelinating polyneuropathy (AIDP) and acute motor axonal neuropathy (AMAN) $)^{2,9,10}$. Patients with AIDP usually have the classic sensorimotor variant of GBS, whereas those with AMAN typically have the pure motor variant ${ }^{8}$. In some patients with axonal GBS, both sensory and motor fibres are affected; this variant is termed acute motor and sensory axonal neuropathy (AMSAN) and is sometimes considered to be a severe variant of $\mathrm{AMAN}^{2}$. Plasma exchange and intravenous immunoglobulin infusions are equally effective therapies for all variants of $\mathrm{GBS}^{2-4,11}$.

Considerable variation between countries and/or regions is evident in the epidemiology, subtypes and management of $\mathrm{GBS}^{12}$. These differences are thought to be related to environmental and economic factors as well as to health awareness and behaviour. Poor hygiene and sanitation, unsafe drinking water and frequent exposure to pathogens render the populations in low-income and middle-income countries (LMIC) defined in July 2019 by the World Bank as countries having an annual gross national income per capita of $<$ US\$3,995 $\left(\right.$ REF. $^{13}$ ) - highly vulnerable to outbreaks of infectious diseases that are capable of triggering $\mathrm{GBS}^{14,15}$. For example, outbreaks of GBS in northern China (2007) and Mexico (2011) were due to increases in the incidence of Campylobacter jejuni infection ${ }^{16,17}$. 


\section{Key points}

- The considerable regional variation evident in the epidemiology, subtypes and management of Guillain-Barré syndrome (GBS) can be explained by geography, population demographics, environmental and economic factors.

- Poor hygiene and sanitation along with frequent exposure to pathogens render populations in low-income and middle-income countries (LMIC) prone to outbreaks of infectious diseases that can trigger GBS.

- High rates of adverse outcomes and mortality in LMIC can be explained by insufficient health-care infrastructure leading to diagnostic delays and lack of available and affordable treatment.

- Owing to differences in disease severity, clinical presentation and patient management between high-income countries (HIC) and LMIC, existing models to predict the outcome of GBS must be validated for LMIC.

- New and low-cost treatment strategies for GBS need to be developed along with improved access to integrative rehabilitation services in LMIC.

Variations in the incidence and outcomes of GBS can also be partly explained by income per capita ${ }^{12,18}$. Resource limitations in LMIC, including the limited availability of electrodiagnostic machines, hospital and intensive care unit (ICU) beds and rehabilitation clinics, can hamper the diagnosis and care of patients with $\mathrm{GBS}^{5}$. In addition, the lack of national guidelines (in most LMIC) and the high cost of treatment facilities complicate the management of patients with GBS versus their counterparts in high-income countries (HIC) - defined according to World Bank criteria as having an annual gross national income per capita of $\geq$ US\$3,995 $\left(\right.$ REF. $\left.^{13}\right)$, which represents the upper middle-income and high-income categories combined ${ }^{19-23}$.

Although the number of studies of GBS in LMIC is increasing, the majority of GBS studies conducted to date have focused on HIC and we are not aware of any prior published reviews focusing on LMIC. Accordingly, this Review aims to provide an overview of GBS in LMIC and to compare the epidemiology, clinical presentation, subtypes and management of GBS in LMIC and HIC. We identify specific challenges related to the diagnosis, treatment and management of patients with GBS in LMIC and explore the prospects for future research and policy.

\section{Epidemiology}

Most studies on the incidence of GBS have been performed in populations from HIC; only a few have included populations from LMIC (TABLE 1). The reported incidence of GBS ranges from 0.16 to 3.0 cases per 100,000 persons/year ${ }^{24,25}$; this considerable variation could,

\footnotetext{
Author addresses

${ }^{1}$ Laboratory of Gut-Brain Signaling, Laboratory Sciences and Services Division, icddr,b, Dhaka, Bangladesh.

2Department of Neurology, Erasmus MC, University Medical Center, Rotterdam, Netherlands.

${ }^{3}$ National Institute of Neurosciences and Hospital, Dhaka, Bangladesh.

${ }^{4}$ Department of Medical Microbiology and Infectious Diseases, Erasmus MC, University

Medical Center, Rotterdam, Netherlands.

${ }^{5}$ Fondation Mérieux, Lyon, France.

${ }^{6}$ Department of Immunology, Erasmus MC, University Medical Center, Rotterdam,

Netherlands.

'These authors contributed equally: Nowshin Papri, Zhahirul Islam.
}

in part, be related to geographical location (FIG. 1). For instance, an incidence of $\sim 0.40$ cases per 100,000 persons/year was reported in Brazil, 0.84-1.91 cases per 100,000 persons/year in Europe and North America and 2.1-3.0 cases per 100,000 persons/year in Iran, Curaçao and Bangladesh ${ }^{2,24-28}$. As well as the factors already mentioned, some of this variation could be due to methodological differences between studies and the lack of robust, systematic population-based studies in certain countries $^{24}$.

Most studies from Europe and North America were performed between 1980 and 2000 and the incidence of GBS in these regions remained stable across most of this period (1.0-1.8 cases per 100,000 persons/year), suggesting a consistent exposure to infectious triggers ${ }^{25}$. Seasonal fluctuations in the incidence of GBS also vary by geographical area. One large meta-analysis showed that the incidence of GBS increases in winter (JanuaryMarch) in western, Middle Eastern and Far Eastern countries, but decreases during January-March in the Indian subcontinent and Latin America ${ }^{29}$. The increased incidence of GBS during winter in some countries is thought to be due to the increased incidence of respiratory tract infections caused by Mycoplasma pneumoniae or Haemophilus influenzae $e^{30,31}$. By contrast, an increase in the incidence of GBS has been observed during summer in northern China and Bangladesh, which is thought to be associated with an increased frequency of preceding diarrhoea ${ }^{3,16,32}$. In these countries, the high temperatures and humidity of the summer season favour bacterial growth and are an important determinant of the burden of bacterial diarrhoea ${ }^{33,34}$.

Almost all reports document a higher incidence of GBS in men than women ( 1.5:1.0), including those from LMIC such as Bangladesh, India, Taiwan, Pakistan, Egypt, Morocco, Ethiopia, Tanzania and Kenya ${ }^{3,4,9,14,23,27,35-43}$. Most studies indicate that the incidence of GBS increases with age, although the age distribution of cases in each country or region is influenced by the demographics of the background population and the number of people in each age group at risk of developing GBS. Thus, in Europe and North America, which have ageing populations, GBS occurs most frequently among people aged $50-80$ years $(2.0-4.0$ cases per 100,000 persons/year $)^{2,24,25}$. By contrast, studies from Asia (Bangladesh, China, India), South America (Brazil) and sub-Saharan Africa (Ethiopia, Tanzania), which are not affected by population ageing, suggest that GBS occurs most frequently in people aged $21-35$ years ${ }^{12,38,40,44,45}$. In LMIC, where Campylobacter infections are endemic, infections are predominantly seen in children, and the rates of Campylobacter-related illness and infection ratios decrease with age ${ }^{46}$. Age can also influence the risk of developing infections that trigger GBS and is an important prognostic factor in individuals with GBS.

The clinical presentation, extent and severity of GBS vary geographically (TABLE 2). In Europe and North America, 90-95\% of patients with GBS have AIDP, and the rest have AMAN or AMSAN ${ }^{7,9,12}$. The proportion of patients with AMAN or AMSAN is considerably higher (30-65\%) in several countries in Latin America, 
Table 1 | Reviewed publications on GBS by region

\begin{tabular}{|c|c|c|c|}
\hline Region & Country & $\begin{array}{l}\text { Number of } \\
\text { studies included }\end{array}$ & Study design (number of patients with GBS per study) \\
\hline \multicolumn{4}{|l|}{ LMIC } \\
\hline East Asia and Pacific & Indonesia & 1 & Retrospective (28) \\
\hline \multirow[t]{2}{*}{ Middle East and North Africa } & Egypt & 4 & Clinical trial (41); cohort $(50,50)$; case-control $(133)$ \\
\hline & Morocco & 1 & Clinical trial (41) \\
\hline \multirow[t]{4}{*}{ South Asia } & India & 14 & $\begin{array}{l}\text { National surveillance programme }(79) \text {; clinical trial }(37,12) \text {; cohort } \\
\left(328,140,102,70^{a}\right) \text {; case-control }(80) \text {; retrospective }(1,166,273 \text {, } \\
173,90) \text {; case reports }(2,1)\end{array}$ \\
\hline & Bangladesh & 10 & $\begin{array}{l}\text { Clinical trial }(20) \text {; cohort }(693,506,407,344,300,300,151) \text {; } \\
\text { case-control }(418,100)\end{array}$ \\
\hline & Pakistan & 3 & Retrospective $(216,175,87)$ \\
\hline & Nepal & 1 & Retrospective (31) \\
\hline \multirow[t]{6}{*}{ Sub-Saharan Africa } & Ethiopia & 1 & Retrospective (95) \\
\hline & Kenya & 1 & Retrospective (54) \\
\hline & Nigeria & 1 & Cohort (34) \\
\hline & Tanzania & 1 & Retrospective (115) \\
\hline & Sudan & 1 & Case report (10) \\
\hline & Zimbabwe & 1 & Cohort (32) \\
\hline \multicolumn{4}{|l|}{ HIC } \\
\hline \multirow[t]{9}{*}{ East Asia and Pacific } & Australia & 2 & Cohort (76); retrospective (46) \\
\hline & China & 6 & Cohort $(541,170,166)$; retrospective $(72)$; case-control $(150,32)$ \\
\hline & Taiwan & 3 & National surveillance programme $(5,998,5,469)$; retrospective $(96)$ \\
\hline & Japan & 2 & Cohort (97); retrospective (40) \\
\hline & French Polynesia & 2 & Case-control (42); national surveillance programme (9) \\
\hline & Thailand & 2 & Retrospective (30); case report (1) \\
\hline & Korea & 1 & National surveillance programme (48) \\
\hline & Singapore & 1 & Retrospective (31) \\
\hline & New Zealand & 1 & National surveillance programme $(2,056)$ \\
\hline \multirow[t]{7}{*}{ Europe and Central Asia } & Netherlands & 4 & Clinical trial $\left(388^{\mathrm{b}}, 85\right)$; retrospective $(67,36)$ \\
\hline & Denmark & 1 & National surveillance programme $(2,319)$ \\
\hline & Germany & 1 & Retrospective (34) \\
\hline & Italy & 1 & Cohort (96) \\
\hline & Norway & 1 & Cohort (52) \\
\hline & Spain & 1 & Retrospective (106) \\
\hline & UK & 1 & Retrospective (110) \\
\hline \multirow[t]{5}{*}{ Latin America and Caribbean } & Brazil & 5 & $\begin{array}{l}\text { National surveyc; cohort }(206,149) \text {; case-control (41); } \\
\text { case report (1) }\end{array}$ \\
\hline & Puerto Rico & 2 & National surveillance programme (56); cohort (123) \\
\hline & Colombia & 1 & Cohort (68) \\
\hline & Curaçao & 1 & Retrospective (49) \\
\hline & Mexico & 1 & National surveillance programme (467) \\
\hline \multirow[t]{2}{*}{ Middle East and North Africa } & Iraq & 1 & National surveillance programme $(2,611)$ \\
\hline & Saudi Arabia & 1 & Retrospective (49) \\
\hline North America & USA & 1 & Case-control (26) \\
\hline South Asia & Sri Lanka & 2 & Case report $(1,1)$ \\
\hline
\end{tabular}

We mainly selected papers published after 1990 (REF.95), but we did not exclude commonly referenced and highly regarded older publications. GBS, Guillain-Barré syndrome; HIC, high-income countries; LMIC, low-income and middle-income countries. ${ }^{2}$ Data were collected prospectively and subjected to retrospective review. 'Data were collected from two randomized controlled trials and one pilot study; a multinational study $(n=10)$; worldwide data, reviews and expert opinion ( $n=30)$. cSurvey responses from Brazilian neurologists (no patients with GBS were included in the survey). 


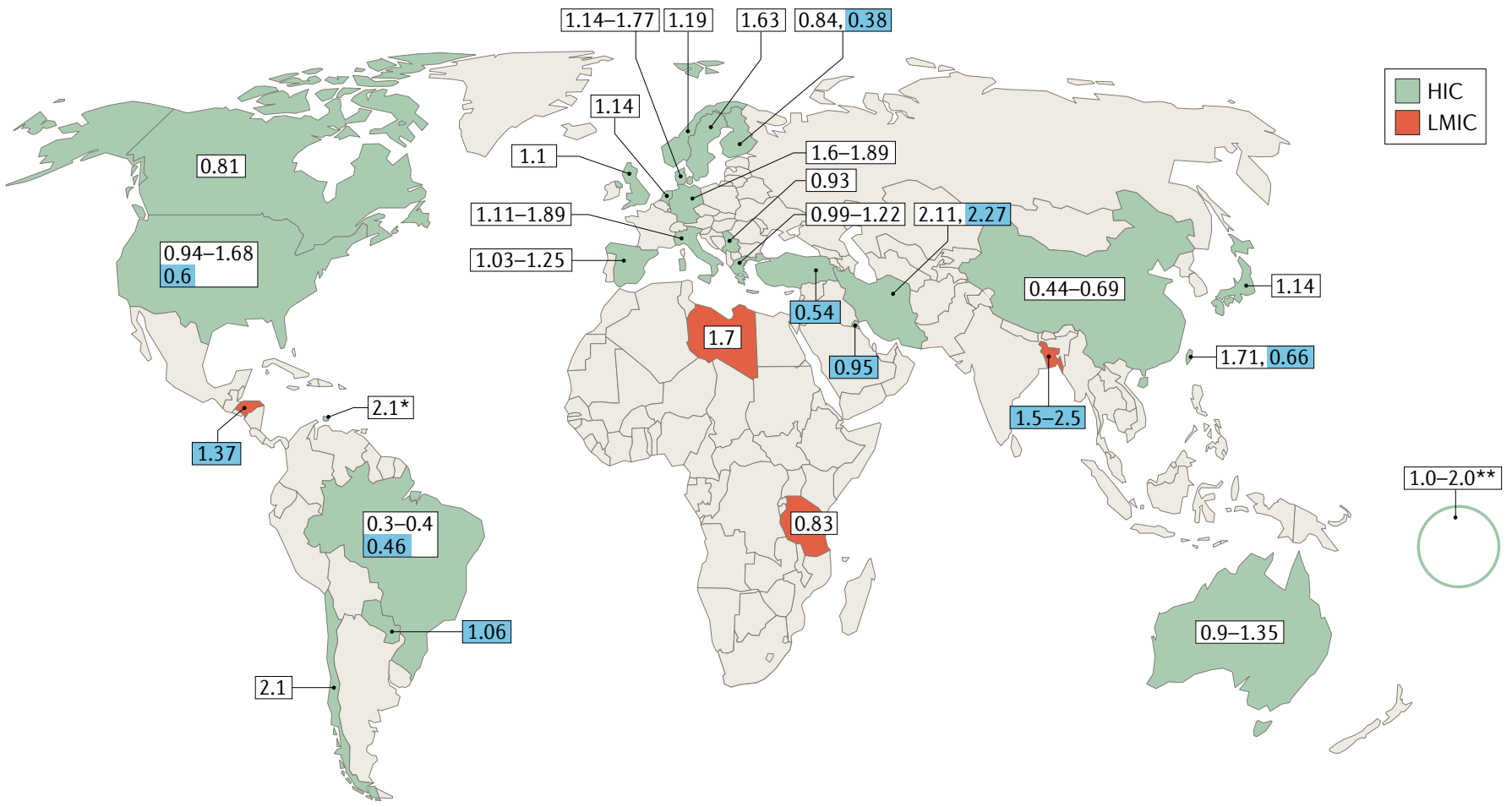

Fig. 1 Reported incidence rates of GBS in HIC and LMIC. According to World Bank definitions, low-income and middleincome countries (LMIC) are those with an annual gross national income per capita of $<$ US $\$ 3,995$, whereas upper middle-income and high-income countries combined (HIC in this Review) have an annual gross national income per capita of $\geq$ US $\$ 3,995$ (REF. ${ }^{13}$ ). Numbers on a white background represent the incidence rate of all cases of Guillain-Barré syndrome (GBS); numbers on a blue background represent the incidence rates of paediatric cases of GBS only. ${ }^{\star} \mathrm{Curaçao,}$ **French Polynesia.

the Caribbean (Curaçao, Mexico, Argentina) and Asia (China, Japan, Bangladesh), although in many of these countries (including Japan) AIDP remains the most frequent variant ${ }^{14,35,44,47-49}$. In the countries and regions where AMAN is the predominant variant, the frequency of AIDP is $22-46 \%{ }^{9}$. MFS seems to be more common among patients with GBS from eastern Asia; as an example, $20-26 \%$ of patients in Taiwan, Singapore and Japan have MFS, a much higher proportion than in the rest of the world $(5-10 \%)^{7,9,50}$. The high prevalence of AMAN, AMSAN and MFS in Asia might be related to the increased frequency of $C$. jejuni infection in this region $^{7,9,14}$. Other infections such as $H$. influenzae have also been linked to MFS in Asia ${ }^{51}$. In countries such as Bangladesh and China, where AMAN is more frequent than it is in Europe and North America, approximately $80 \%$ of patients present with severe GBS (GBS disability score $>2$ ) compared with $40-60 \%$ of patients from Europe and North America, where the AIDP subtype is most prevalent ${ }^{12,52}$.

\section{Pathogenesis}

Overall, GBS is considered to be the consequence of a preceding infection that triggers an immune response that is responsible for the demyelination and axonal degeneration of peripheral nerves and nerve roots. Treatment with immunomodulatory agents, such as vaccines or biologic drugs, have also been associated with GBS in rare individuals. Other events, including surgery and malignancy, have been temporally related to GBS; the underlying mechanism of GBS in such individuals is not clear ${ }^{53-55}$.

Antecedent infections. Approximately two thirds of patients with GBS report symptoms of an infectious disease within the 4 weeks preceding the onset of weakness $^{2}$. Upper respiratory tract infection is the most common antecedent event and is reported by $22-53 \%$ of all patients with GBS in Europe, North America, South America and parts of Asia (Taiwan, Nepal, Pakistan, Japan and Malaysia) $)^{10,12,37,47,56}$. The frequency of antecedent respiratory tract infections is even higher in paediatric patients with GBS (50-70\%) $)^{25}$. By contrast, in India and Bangladesh, gastroenteritis is the most frequent antecedent event associated with GBS (36-47\%) $)^{12,57}$.

Worldwide, the most frequently identified infectious agent that triggers GBS is C. jejuni, which is an important bacterial cause of gastroenteritis and food poisoning ${ }^{30,58}$. The reported frequencies of antecedent C. jejuni infection in patients with GBS differ between studies as well as between countries and regions; for instance, C. jejuni infection is substantially more frequent among patients with GBS from Curaçao, China and Bangladesh ( $60-70 \%)$ than in those from all other countries $(30-32 \%)^{14,28,32,48}$. The increased frequency of C. jejuni infection in these regions could be explained by their hygienic infrastructure and environmental or host-related factors, including $\operatorname{diet}^{14,27,44,59}$. C. jejuni is an established cause of MFS that is probably more frequent in $\mathrm{LMIC}^{60}$. However, other infections might 
be responsible for triggering MFS in countries where C. jejuni is less common.

The reported frequencies of antecedent infections in a given population can change over time. For example, China has undergone rapid socioeconomic development and improvements in health services over the past 50 years. A recent study of GBS in China found a lower incidence of antecedent $C$. jejuni infection (27\% in data from $2013-2017)^{59}$ than had previously been reported $(66 \% \text { in data from 1991-1992) })^{32}$. In addition, the trend towards increased life expectancy in China over a similar time period could have decreased the incidence of $C$. jejuni infections, which are more common in younger individuals. We are not aware of any public health interventions undertaken during this time by the Chinese government aimed specifically at reducing the number of $C$. jejuni infections ${ }^{59,61}$. However, public health interventions can both reduce the prevalence of Campylobacter infections and decrease the incidence of GBS: in response to high rates of $C$. jejuni infection between 1980 and 2006, the New Zealand government introduced a national intervention to reduce contamination with Campylobacter spp. in poultry. Within 2 years, the country achieved a $52 \%$ decline in campylobacteriosis and a simultaneous 13\% reduction in GBS hospital admissions ${ }^{62}$. Whether such infection control interventions are feasible in other countries and regions (such as LMIC) remains to be fully explored.

Other infectious agents that have been detected at higher frequencies in patients with GBS than in the background population are cytomegalovirus (10-13\%), EpsteinBarr virus (10\%), M. pneumoniae (5\%; predominantly

\section{Table 2 | Clinical features and outcome of GBS by region}

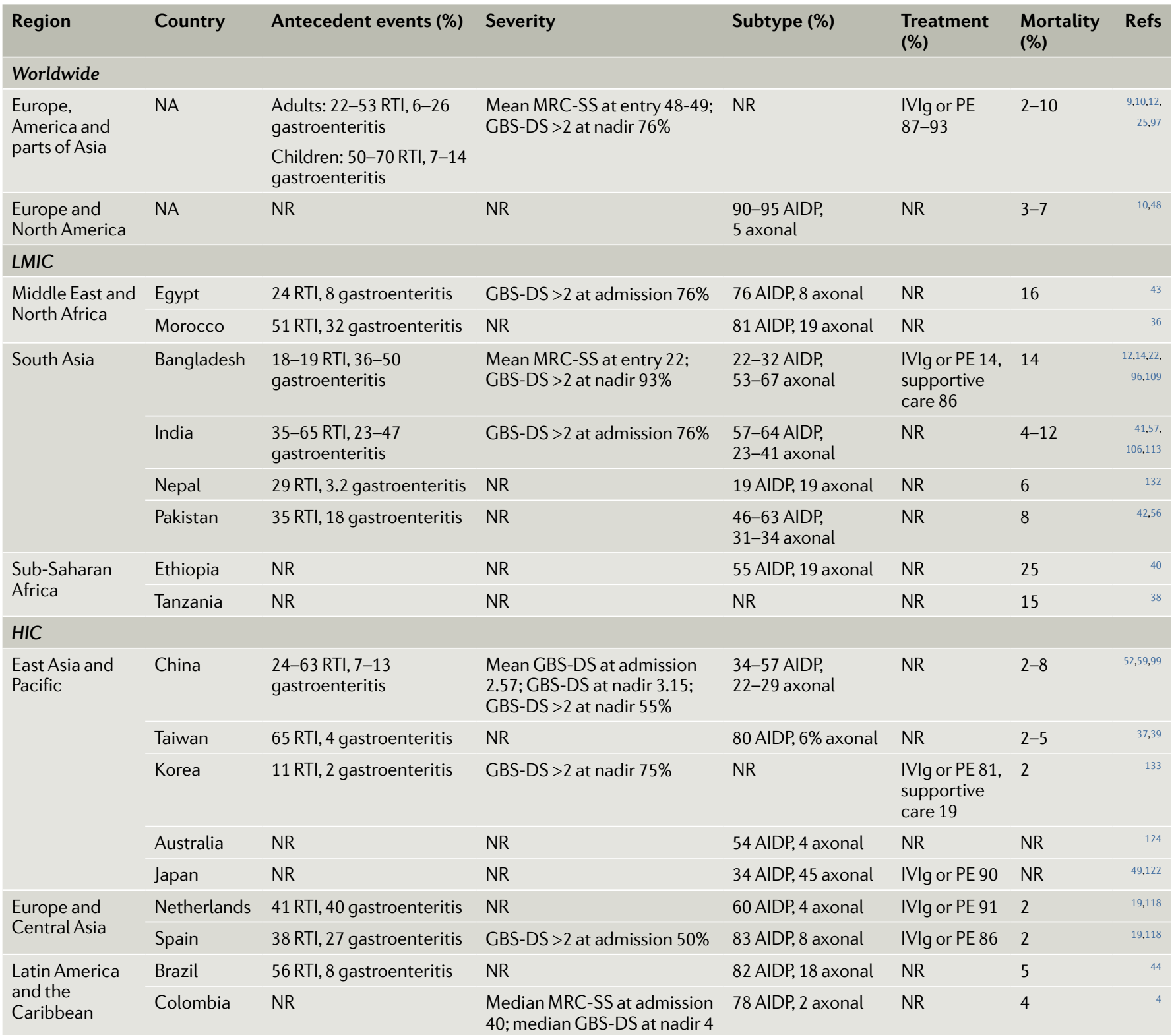

AIDP, acute inflammatory demyelinating polyneuropathy; GBS-DS, Guillain-Barré syndrome disability score; HIC, high-income countries; IVIg, intravenous immunoglobulin; LMIC, low-income and middle-income countries; NA, not applicable; NR, not reported; MRC-SS, Medical Research Council sum score; PE, plasma exchange, RTI, respiratory tract infection. 
in children), hepatitis E virus (5\%) and Zika virus ${ }^{30,48,58,63}$. Additionally, some infections that are more frequent in LMIC than in other countries and regions have been associated with GBS in case reports or case series: malaria in India, Sri Lanka and Thailand; HIV infection in sub-Saharan Africa; and dengue virus infection in Southeast Asia and Brazil ${ }^{64-70}$. To our knowledge, no reports have linked these infections to GBS in HIC, and epidemiological or case-control studies are required to confirm whether these infections are truly associated with GBS. During the coronavirus disease 2019 (COVID-19) pandemic, several case reports or case series have indicated a possible association between GBS and severe acute respiratory syndrome coronavirus 2 (SARS-CoV-2) infection ${ }^{71-74}$. At the time of writing, most such reports were from Europe, although a small number of case reports were from LMIC (four from India, one from Morocco and one from Sudan $)^{75-77}$. However, an epidemiological study in the UK found no increase in the incidence of GBS during the COVID-19 pandemic ${ }^{78}$. Further studies are required to confirm the potential relationship between SARS-CoV-2 infection and GBS.

Immunopathogenesis. The geographic differences in clinical and electrophysiological phenotypes of GBS in LMIC and HIC might be in part caused by differences in the rates of preceding infections that tend to trigger different types of GBS. For example, $C$. jejuni infections lead to the development of predominantly (but not exclusively) the axonal type of $\mathrm{GBS}^{30}$. In C. jejuni-related GBS, an immune response is triggered owing to molecular mimicry between $C$. jejuni lipo-oligosaccharides and human nerve gangliosides, which results in the production of cross-reactive antibodies that activate complement and damage nerves ${ }^{2}$. The pathophysiological mechanisms leading to GBS after infections other than C. jejuni have not yet been clearly defined, but similar mechanisms might also play a part in other bacterial infections related to GBS, such as M. pneumoniae and $H$. influenzae, although these have been less extensively investigated.

The demyelinating and sensorimotor forms of GBS are usually preceded by infection with viruses, such as cytomegalovirus or Epstein-Barr virus; however, the immunopathogenesis remains to be elucidated ${ }^{10}$. Similarly, the specific components of the Zika virus that trigger the immune response leading to GBS have not yet been clarified ${ }^{79}$. Of the patients with SARS-CoV-2associated GBS, 77-80\% had the demyelinating electrophysiological subtype and $\sim 70 \%$ had classic sensorimotor $\mathrm{GBS}^{80,81}$. Whether this is the typical phenotype of SARS-CoV-2-related GBS is presently unclear owing to the limited number of reported cases.

Despite the strong associations between specific infectious agents and GBS, the overall risk of developing GBS after infection is very small; for example, only one in 1,000-5,000 patients with $C$. jejuni infection will develop GBS in the subsequent 2 months. One factor that determines this low risk is the requirement for carbohydrate mimicry (which is not present in all C. jejuni strains) to develop the cross-reactive antibody response to gangliosides that evolves into $\mathrm{GBS}^{2,3,10}$. However, genetic and nutritional factors might also influence the patient's susceptibility to producing such antibodies ${ }^{82-84}$. Poor nutritional status, and specifically malnutrition, alters the dysfunctional immune responses implicated in the pathogenesis of various autoimmune diseases ${ }^{85}$. Immune response activation following an infection has also been associated with genetic polymorphisms. Several studies have found associations between GBS and polymorphisms in the TNF gene (which encodes tumour necrosis factor) and the $M B L 2$ gene (which encodes mannose-binding protein $C)^{2,82-84}$.

Outbreaks of GBS. Although GBS usually occurs sporadically, several outbreaks of this disease have been linked to epidemics of infectious diseases that can trigger GBS. Surges in GBS cases in China (2007) and Mexico (2011) were linked to epidemics of $C$. jejuni infection, and an outbreak of GBS in Peru in 2018 was associated with an epidemic of enterovirus infection ${ }^{16,17,86}$. A link between GBS and Zika virus infection was first reported when a 20-fold increase in GBS cases was found during a Zika virus outbreak in French Polynesia in 2013-2014. Subsequently, the incidence of GBS rose by $\sim 3.2-5.1$ times in areas affected by the Zika virus epidemic in Latin America and the Caribbean (2014-2016) ${ }^{4-6,17,63,87}$. However, only $\sim 2$ in 10,000 patients infected with Zika virus went on to develop GBS, suggesting that a relatively large outbreak of Zika virus is necessary to increase the incidence of $\mathrm{GBS}^{88}$.

The origins of emerging infectious diseases correlate positively with specific socioeconomic, environmental and ecological factors, which provide a basis for identifying regions where new infections are most likely to originate (so-called emerging disease hotspots) ${ }^{89}$. Zoonoses from wildlife represent the most important and growing threat of emerging infections to global health, whereas vector-borne diseases are responsible for about $25 \%$ of emerging infectious diseases. Hotspots for emerging infectious diseases are more common at lower latitudes where wild animals and arthropod vectors reside, such as sub-Saharan Africa and parts of Asia, which mainly consist of LMIC $^{89}$. Other vector-borne viruses transmitted by the same Aedes family of mosquitoes as Zika virus (such as chikungunya and dengue) have also been associated with surges in GBS cases ${ }^{90,91}$. Therefore, these regions are particularly at risk of new outbreaks of GBS. In response to the Zika virus outbreak, several projects have been set up in Latin America to prevent transmission of vector-borne diseases, including surveillance systems for arboviruses and vector control programmes ${ }^{92}$. Further investment in these projects and their implementation in at-risk areas beyond Latin America could help to reduce the likelihood of future outbreaks of GBS.

International disease surveillance initiatives could also help identify new outbreaks of GBS. The ongoing acute flaccid paralysis (AFP) surveillance programme originally devised for the surveillance of poliomyelitis - is a useful early warning signal that flags changes in the prevalence of AFP in children up to 15 years of age. Studies conducted in China and Bangladesh show that GBS is now the predominant cause of AFP among 
children in this age group, suggesting that AFP surveillance programmes could be expanded to detect changes in the incidence of GBS. Data from this programme have already been used to calculate crude incidence rates of GBS among children ${ }^{26,93}$. Extending the AFP surveillance programme to other age groups, and GBS case ascertainment using the Brighton Collaboration criteria to assess the degree of diagnostic certainty, might help to monitor the incidence of $\mathrm{GBS}^{94}$.

\section{Diagnosis}

Diagnosis of GBS is mainly based on clinical features, supported by cerebrospinal fluid examination and nerve conduction studies. The National Institute of Neurological Disorders and Stroke (NINDS) criteria and the Brighton Collaboration criteria are the most commonly used sets of validated diagnostic criteria for GBS $^{1-3,19,95-99}$.

Patients with GBS can present with remarkably diverse clinical features. In patients with typical GBS, the key presenting feature is ascending bilateral symmetrical weakness that progresses over a period of $12 \mathrm{~h}$ to 28 days before a plateau is reached ${ }^{1-3,9,10}$. Most patients develop generalized hyporeflexia or areflexia, although tendon reflexes can be normal or even exaggerated in the initial stages. More than half of patients with GBS develop cranial nerve deficits, including bilateral facial weakness, bulbar weakness or extraocular motor dysfunction. In addition to muscle weakness, patients can also experience sensory disturbances, ataxia, muscle pain or radicular pain and signs of autonomic dysfunction, including blood pressure fluctuations and cardiac arrhythmia $^{1-3,12}$. This diversity can complicate diagnosis in the early stages of GBS, especially in patients with atypical findings - for instance, the $\sim 10 \%$ of patients who have normal or brisk deep tendon reflexes and the $\sim 8 \%$ of patients who present with only paraparesis ${ }^{100}$. Children with GBS might also present with atypical features such as pain, refusal to walk or an abnormal gait; indeed, GBS is correctly diagnosed at admission in only one-third of affected preschool-aged children ${ }^{2}$. Diagnosis is generally even more challenging in LMIC, where facilities for cerebrospinal fluid examination and nerve conduction studies might not be readily available, which leads to multiple referrals of patients and diagnostic delay. In one prospective multinational cohort study, the median interval between the onset of weakness and study entry was 5 days in the Netherlands compared with 10 days in Bangladesh ${ }^{96}$. Studies conducted in Africa have also found lengthy intervals of up to 19 days between the onset of weakness and hospitalization ${ }^{38}$. This delay could lead to under-reporting of GBS in LMIC, as some patients with severe disease might die before reaching the hospital. Moreover, patients with mild symptoms might not seek treatment or recover before reaching a hospital.

The relationships between $C$. jejuni infection and antibodies against the GM1, GM1b, GD1a, GalNAcGD1a and GQ1b gangliosides in patients with GBS are well established ${ }^{2}$. Some studies have suggested an association between the presence of anti-GM2 antibodies and a recent cytomegalovirus or Epstein-Barr virus infection $^{59,101}$. However, serological tests to detect antiganglioside antibodies are not routinely performed at diagnosis, as negative test findings cannot rule out $\mathrm{GBS}^{2}$. Furthermore, most of these serological tests require sophisticated techniques and trained personnel that might not be available in LMICs.

In addition, an extensive list of differential diagnoses might need to be excluded. The differential diagnosis of GBS depends on the clinical presentation and variant of GBS (BOX 1) and is also likely to differ between countries and regions, owing to local variations in the prevalence of infectious diseases, nutritional deficiencies or intoxications, autoimmune diseases and malignancies. As no region-specific information on the differential diagnosis of GBS was included in published studies, we conducted a small survey (Supplementary Information) to obtain insight into this important characteristic. The survey was sent to GBS experts working in LMIC within our network, who were asked in turn to distribute the questionnaire to other neurologists in their networks. In total, 17 neurologists (of whom seven frequently see paediatric patients) and two paediatric neurologists working in LMIC returned the questionnaire. Their responses revealed that the differential diagnosis of GBS is generally comparable between LMIC and HIC, although some important differences were noted (N.P., S.E.L., Q.D.M. and B.C.J., unpublished observations). For example, sarcoidosis, Sjögren syndrome, LambertEaton myasthenic syndrome and mitochondrial disease seem to be less frequent diagnoses among patients suspected of GBS in LMIC than in HIC. Other diagnoses, such as hypokalaemic thyrotoxic periodic paralysis, organophosphate intoxication, botulism, rabies, polio and tetanus, seem to be more frequent in LMIC than in HIC. Furthermore, the infectious causes of transverse myelitis, acute flaccid myelitis and polyradiculoneuritis differ between LMIC and HIC. Lyme borreliosis and enterovirus D68 or A71 infection are rarely seen outside Europe and North America, whereas infections with HIV, HTLV-1 and arthropod-borne viruses - including Zika virus, chikungunya virus and West Nile virus are frequently reported in several LMIC. These differences might reflect geographic variation in the spread of arthropod vectors (such as those carrying arboviruses) or in the incidence of infectious diseases. For example, polio and rabies eradication programmes have been more successful in HIC than in LMIC. Other explanations might include resource limitations in LMIC that preclude the diagnosis of complex systemic disorders such as Sjögren syndrome and differences in the ages of the populations at risk.

The differential diagnosis of paediatric GBS differs from that in adults owing to the presence of atypical or non-specific features that complicate the diagnosis, such as meningism or poorly localized pain ${ }^{102,103}$. Furthermore, vascular causes, vitamin deficiencies, drug-induced myopathy or polyneuropathy and chronic inflammatory demyelinating polyneuropathy occur less frequently in children than in adults ${ }^{102,103}$. These differences between adults and children in the differential diagnosis of GBS occur in both HIC and LMIC, although (as reported for adults) the infectious causes 


\section{Box 1 | Differential diagnosis of GBS}

\author{
Infection \\ - Acute transverse myelitis (associated with HIV, cytomegalovirus, Epstein-Barr virus, \\ varicella zoster virus, syphilis, tuberculosis or diphtheria infection) \\ - Acute flaccid myelitis due to infections with arthropod-borne viruses (such as Zika \\ virus, chikungunya virus, West Nile virus) or other viruses such as rabies, polio and \\ enterovirus D68 or A71 \\ - Poly(radiculo)neuritis owing to infection with HIV, cytomegalovirus, Epstein-Barr virus, \\ varicella zoster virus, diphtheria or Lyme borreliosis \\ - Botulism (Clostridium botulinum) or tetanus (Cl. tetani) \\ - Myositis caused by influenza virus, HIV, HTLV-1 or enterovirus infection ${ }^{\mathrm{b}}$ \\ - Meningitis and/or meningoencephalitis ${ }^{\mathrm{b}}$

\section{Inflammation} \\ - Acute transverse myelitis \\ - Neuromyelitis optica, myelin oligodendrocyte glycoprotein antibody-associated \\ disorder, sarcoidosis, Sjögren syndrome \\ - (Acute onset) chronic inflammatory demyelinating polyneuropathy (CIDP) \\ - Myasthenia gravis \\ - Lambert-Eaton myasthenic syndrome
}

\section{Metabolic}

- Electrolyte disorders such as hypokalaemia or hypokalaemic thyrotoxic periodic paralysis (common), hypophosphataemia or hypermagnesaemia

- Deficiency of vitamin $B_{1}$ (associated with beriberi or Wernicke's encephalopathy), vitamin $B_{12}$ (associated with subacute combined degeneration of the spinal cord) and vitamin $\mathrm{E}^{\mathrm{c}}$

- Porphyria

- Diabetic neuropathy and/or drug-induced diabetic neuropathy ${ }^{c}$

- Hyperthyroidism and hypothyroidism

- Copper deficiency

\section{Malignancy}

- Leptomeningeal metastases or neurolymphomatosis

- Brainstem or spinal cord tumour

\section{Vascular}

- Brainstem or spinal cord stroke

- Vasculitis

\section{Toxins}

- Organophosphates (common), lead, thallium, arsenic, diethylene glycol, ethylene glycol, methyl alcohol (methanol) and $\mathrm{N}$-hexane

- Ethyl alcohol (ethanol) or paraquat poisoning

- Drug-induced (for example, by colchicine, chloroquine, emetine or statins)

- Snakebite envenomation

\section{Mechanical factors}

- Compression of the brainstem or spinal cord ${ }^{\mathrm{b}}$

- Cauda equina syndrome

\section{Other}

- Functional and/or conversion disorder

- Critical illness polyneuropathy

- Myopathy or acute rhabdomyolysis

- Mitochondrial disease

GBS, Guillain-Barré syndrome. a Polio has been eradicated in most regions, with the exception of several countries in sub-Saharan Africa and Southeast Asia (mostly Pakistan), where sporadic cases can occur. Although this box mainly focuses on the differential diagnosis of GBS in adults, ${ }^{b}$ diagnoses that are more common in children than in adults, and ${ }^{c}$ diagnoses that are less common in children than in adults are indicated. of conditions that mimic paediatric GBS differ between LMIC and HIC.

\section{Treatment}

Management of GBS requires a multidisciplinary approach including supportive medical care and immunotherapy. Intravenous immunoglobulin $(0.4 \mathrm{~g} / \mathrm{kg}$ for 5 days) and plasma exchange (usually five sessions at $200-250 \mathrm{ml} / \mathrm{kg}$ ) are proven and equally effective treatments for $\mathrm{GBS}^{3,11,104}$. However, most randomized controlled trials that evaluated the effectiveness of these two treatments for GBS were conducted in populations from HIC. These trials mainly included adult patients who were treated either with intravenous immunoglobulin within 2 weeks or with plasma exchange within 4 weeks after the onset of weakness ${ }^{11,104}$. Included patients had a GBS disability score of $\geq 3$ and the majority had the AIDP subtype of GBS ${ }^{11,104}$. Therefore, the efficacy of these therapies might differ in LMIC, where AMAN and AMSAN are prevalent and patients usually present to hospital in the later stages of disease than they do in HIC.

Considerable variations in treatment protocols for GBS are observed throughout the world ${ }^{21}$. In general, intravenous immunoglobulin is considered the first choice of treatment as it is easy to administer, widely available and associated with a reduced frequency of adverse effects compared with plasma exchange ${ }^{11,105}$. Conversely, plasma exchange is less costly than intravenous immunoglobulin and could theoretically be a preferred treatment option for GBS in $\mathrm{LMIC}^{106-108}$. However, in practice, clinicians in LMIC face various limitations and obstacles that were not considered in existing GBS therapeutic studies. For example, owing to the low per capita income and lack of coverage by the national health insurance system in Bangladesh, neither intravenous immunoglobulin ( US\$12,000-16,000) nor plasma exchange ( US\$4,500-5,000) are affordable for the majority of patients ${ }^{18}$. Therefore, only $10-12 \%$ of patients in Bangladesh receive one of these treatments, even though most patients with GBS in Bangladesh are severely affected. For instance, $93 \%$ of patients from Bangladesh were unable to walk independently at nadir (GBS disability score $>2$ ) in comparison with $76 \%$ of patients in Europe, America or other parts of Asia $^{12,18,22,109}$. This situation underscores the need for low-cost and effective treatment strategies for GBS in LMIC. Small volume plasma exchange (SVPE) is a novel, relatively low cost $(\sim 500)$, simple technique for selective removal of plasma, and has been shown to be a safe and feasible treatment for GBS in resource-limited settings such as India and Bangladesh ${ }^{18,110}$. However, as the efficacy of SVPE has only been shown in a small number of patients, large-scale studies are required before this technique can be implemented in routine clinical practice.

Complement inhibitors are a new focus in the treatment of GBS in HIC. Eculizumab, a humanized monoclonal recombinant antibody against complement factor 5 , is currently being studied in the UK and Japan ${ }^{71,105}$. Another humanized antibody against complement factor 3 was shown to be safe and well tolerated in patients with 
$\mathrm{GBS}^{111}$, and efficacy trials of this agent are currently ongoing in Europe, the USA and Asia. Although the high cost of these biologic agents is likely to greatly restrict their use in patients with GBS from LMIC, such drugs might be made available for specific indications within LMIC at affordable price levels in the future; for instance, HIV drugs have been made available to some African countries at much lower prices than in HIC ${ }^{112}$. Moreover, several different phases of efficacy trials for complement factor 3 inhibitors are currently ongoing in patients in Bangladesh, which indicates that research groups in some LMICs are able to conduct treatment trials in accordance with the latest scientific methods and regulatory requirements. We hope that this experience will lead to opportunities to develop affordable treatments for patients with GBS in LMIC in future.

\section{Outcome and prognosis}

Admission to the ICU is recommended for patients with GBS who have imminent respiratory insufficiency, severe autonomic dysfunction with cardiovascular instability, severe swallowing dysfunction and/or diminished cough reflex or rapidly progressive weakness ${ }^{109,113-116}$. However, in LMIC the number of ICU beds is limited and ICU services in private hospitals are too costly ( US\$300-1,200 daily) for most patients ${ }^{71,117}$. A study from Bangladesh found that the absence of ICU support when required was the strongest risk factor for death in patients with $\mathrm{GBS}^{22}$.

In most studies worldwide, the mortality rate for GBS is $2-10 \%{ }^{9,10,97}$ although disparities are evident between regions. For example, reported mortality rates are 2-7\% in Europe and North America ${ }^{10,12,19,118}, 13 \%$ in Hong Kong $^{42}, 14-17 \%$ in Bangladesh ${ }^{12,14,22}$ and $16 \%$ in Egypt ${ }^{119}$. Moreover, access to integrative rehabilitation services is limited in LMIC, which can adversely affect recovery and long-term quality of life of patients with $\mathrm{GBS}^{120}$. Across the globe, $\sim 20 \%$ of patients with GBS are unable to walk unaided 6 months after disease onset ${ }^{2,3,9,10,97,121}$ and this rate is higher (30-40\%) in countries such as Bangladesh where AMAN predominates and most patients do not receive immunotherapy ${ }^{12,14,122}$. In addition to physical complications, a substantial proportion of patients in HIC experience residual problems, including persistent pain $(\sim 35-40 \%)$, fatigue $(60-80 \%)$ and anxiety or depression $(6-7 \%)^{2,123,124}$. No data have been reported on rates of these complications in LMIC. However, as most patients with GBS in LMIC only receive supportive care, these sequelae are also likely to vary between countries and to be worse in patients in LMIC than in those in HIC.

The ability to predict which patients with GBS will develop respiratory insufficiency or have a poor prognosis has been a long-held desire worldwide, as it would enable physicians to take the necessary precautions and provide additional treatment for the patients most at risk ${ }^{47,125}$. To this end, the Erasmus GBS Respiratory Insufficiency Score (EGRIS) was developed to predict the risk of requiring mechanical ventilation within 1 week and the Erasmus GBS Outcome Score (EGOS) and modified EGOS (mEGOS) were developed to predict the outcomes in patients with GBS at 6 months ${ }^{47,116,125}$. However, these tools were derived and validated in cohorts from European countries and might not be applicable worldwide. Indeed, a study from northeast Brazil found that EGOS was not a good predictive tool in that population ${ }^{126}$. By contrast, both EGRIS and mEGOS can accurately predict GBS outcome in populations from Japan and Malaysia ${ }^{127,128}$. Therefore, these models might need to be validated or adapted before they can be used in LMIC.

Various measures have been employed to capture outcomes in clinical trials of GBS around the world. Improvement in GBS disability scale scores is the main prognostic variable in the majority of studies. The Rasch-built Overall Disability Score (RODS), Overall Neuropathy Limitations Scale (ONLS), and Fatigue Severity Scale (FSS) were developed as outcome measures for clinical trials and are used to assess disability, activity limitations and fatigue, respectively, in patients with GBS ${ }^{129-131}$. However, these tools were developed in cohorts of patients with GBS from HIC and the questions might not be culturally appropriate in LMIC.

\section{Conclusions and future prospects}

At present, only limited data are available on GBS in LMIC. Most studies in LMIC were conducted in South Asia (Bangladesh and India) and publications from other LMIC are scarce, especially from Africa. LMIC are hotspots for many emerging infectious disease outbreaks, some of which have been associated with GBS. Therefore, publications from LMIC are often related to outbreaks of GBS associated with specific antecedent infectious diseases. Owing to the lack of well-designed epidemiological studies, the true incidence of GBS in many LMIC remains largely unknown. The long intervals between onset of weakness and hospitalization that are frequently observed in patients in LMIC might introduce selection bias at the hospital level, as patients with mild symptoms might not reach the health system and severely affected individuals might die before reaching the hospital. Moreover, diagnostic facilities, health-care infrastructure and adequately trained health professionals are frequently lacking in LMIC. The absence of national treatment guidelines and high costs of existing treatments relative to local wages contribute to the worse outcomes and higher mortality rates of GBS in LMIC compared with HIC. Finally, current models for predicting the outcome of GBS might not be valid in LMIC, owing to variations in disease severity, clinical presentation, electrophysiological subtypes and management.

A number of strategies can address these challenges. Firstly, the expansion and improvement of GBS research capacity in LMIC is required. Systematic population-based surveillance and cohort studies that employ accurate standardized case definitions are needed to understand and monitor the incidence and overall burden of GBS. Case-control studies are crucial to identify the risk factors associated with GBS and to detect new antecedent infections that trigger GBS in LMIC. Observational cohort studies are important to define the clinical course of GBS and the factors that influence and predict this course. An example of a cohort study of GBS that is ongoing globally in both 
LMIC and HIC is the International GBS Outcome Study $(\text { IGOS })^{96}$. The standardized trial protocol and web-based data entry system used in this international prospective cohort study are an example of how methodological differences between GBS studies conducted in different regions and countries might be overcome. However, African and Latin American countries and regions are under-represented in IGOS, and expanding the study to these regions and the long-term sustainability of this global initiative needs to be assured. Nonetheless, IGOS has already highlighted differences in the presentation and outcome of patients with GBS between HIC and LMIC such as Bangladesh, which provide insight into the challenges associated with caring for these patients in LMIC that might facilitate future research ${ }^{12}$.

Affordable and cost-effective treatment strategies need to be developed and multinational efficacy trials are required to study and scale-up innovative treatment approaches. Several randomized controlled trials, including a safety, feasibility and efficacy trial of SVPE and a phase I (leading to phase II-III) trial of a new investigational drug are currently being conducted in Asia, Europe and the USA. Additional clinical intervention studies of innovative and affordable treatments need to be designed, taking into account the specific context of the health system challenges in LMIC. A sustainable clinical trial infrastructure including physical health-care facilities and adequately trained health professionals needs to be established to support research into GBS in LMIC; these efforts should include high-quality diagnostic laboratories and training programmes for health-care professionals involved in the management of patients with GBS and in clinical research.

Moreover, existing prognostic models need to be validated and adapted for use in LMIC. Such tools would help clinicians in LMIC to accurately identify the patients most in need of ICU care at an early stage, thereby improving the management of individual patients and increasing the efficiency of ICU services in low-resource settings. Valid, responsive and cross-culturally applicable outcome measures need to be developed to improve our understanding of the long-term outcome of GBS in LMIC and to optimize the management of patients in rehabilitation services. Patients and their caregivers can also contact the GBS|CIDP Foundation International for support.

In conclusion, GBS is an under-reported disease in LMIC, although the limited available evidence suggests that the disease has a more severe clinical course in LMIC and that affected patients in LMIC have worse outcomes than do their counterparts in HIC. This Review highlights the most important knowledge gaps and provides suggestions and recommendations for future research. Increasing the breadth and quality of fundamental and applied research should become a critical focus to improve the clinical management of GBS in LMIC in the future. More than 100 years after the first description of the syndrome by George Guillain, Jean Alexandre Barré and André Strohl, now is the time to reduce the disease burden of GBS in LMIC.

Published online 1 March 2021
1. Fokke, C. et al. Diagnosis of Guillain-Barré syndrome and validation of Brighton criteria. Brain 137, 33-43 (2014).

2. Van Den Berg, B. et al. Guillain-Barré syndrome: pathogenesis, diagnosis, treatment and prognosis. Nat. Rev. Neurol. 10, 469 (2014).

3. Van Doorn, P. A., Ruts, L. \& Jacobs, B. C. Clinical features, pathogenesis, and treatment of GuillainBarrê syndrome. Lancet Neurol. 7, 939-950 (2008).

4. Parra, B. et al. Guillain-Barrê syndrome associated with Zika virus infection in Colombia. N. Engl. J. Med. 375, 1513-1523 (2016).

5. Leonhard, S. E., Conde, R. M., de Assis Aquino Gondim, F. \& Jacobs, B. C. Diagnosis and treatment of Guillain-Barré syndrome during the Zika virus epidemic in Brazil: a national survey study. J. Peripher. Nerv. Syst. 24, 340-347 (2019).

6. Cao-Lormeau, V.-M. et al. Guillain-Barré syndrome outbreak associated with Zika virus infection in French Polynesia: a case-control study. Lancet 387 1531-1539 (2016)

7. Dimachkie, M. M. ¿ Barohn, R. J. Guillain-Barré syndrome and variants. Neurol. Clin. 31, 491-510 (2013).

8. Hosokawa, T. et al. Serial electrophysiological findings in Guillain-Barré syndrome not fulfilling AIDP or AMAN criteria. J. Neurol. 263, 1709-1718 (2016).

9. Yuki, N. \& Hartung, H.-P. Guillain-Barré syndrome. N. Engl. J. Med. 366, 2294-2304 (2012).

10. Willison, H. J., Jacobs, B. C. \& Van Doorn, P. A Guillain-Barrê syndrome. Lancet 388, 717-727 (2016).

11. Hughes, R. A. et al. Immunotherapy for Guillain-Barré syndrome: a systematic review. Brain 130 2245-2257 (2007)

12. Doets, A. Y. et al. Regional variation of Guillain-Barré syndrome. Brain 141, 2866-2877 (2018).

13. The World Bank. Data: World Bank Country and Lending Groups. The World Bank http://databank. worldbank.org/data/download/site-content/OGHIST.xIs (2019).

14. Islam, Z. et al. Axonal variant of Guillain-Barré syndrome associated with Campylobacter infection in Bangladesh. Neurology 74, 581-587 (2010).
15. Pal, M., Ayele, Y., Hadush, M., Panigrahi, S. \& Jadhav, V. J. Public health hazards due to unsafe drinking water. Air Water Borne Dis. 7, 1000138 (2018).

16. Zhang, M. et al. Association study between an outbreak of Guillain-Barré syndrome in Jilin, China, and preceding Campylobacter jejuni infection. Foodborne Pathog. Dis. 7, 913-919 (2010).

17. Jackson, B. et al. Binational outbreak of GuillainBarré syndrome associated with Campylobacter jejun infection, Mexico and USA, 2011. Epidemiol. Infect. 142, 1089-1099 (2014).

18. Islam, B. et al. Small volume plasma exchange for Guillain-Barré syndrome in resource-limited settings: a phase II safety and feasibility study. BMJ Open 8 e022862 (2018)

19. Roodbol, J. et al. Diagnosis of Guillain-Barré syndrome in children and validation of the Brighton criteria. J. Neurol. 264, 856-861 (2017).

20. Van der Meché, F. Van Doorn, P. Meulstee, J \& Jennekens, F. Diagnostic and classification criteria for the Guillain-Barré syndrome. Eur. Neurol. 45 133-139 (2001).

21. Verboon, C. et al. Current treatment practice of GuillainBarré syndrome. Neurology 93, e59-e76 (2019).

22. Ishaque, T. et al. High mortality from Guillain-Barré syndrome in Bangladesh. J. Peripher. Nerv. Syst. 22, 121-126 (2017)

23. Nagappa, M. et al. Guillain-Barré syndrome in the elderly: experience from a tertiary-care hospital in India. J. Clin. Neurosci. 46, 45-49 (2017)

24. Sejvar, J. J., Baughman, A. L., Wise, M. \& Morgan, O. W. Population incidence of Guillain-Barré syndrome: a systematic review and meta-analysis. Neuroepidemiology 36, 123-133 (2011).

25. McGrogan, A., Madle, G. C., Seaman, H. E. \& De Vries, C. S. The epidemiology of Guillain-Barre syndrome worldwide. Neuroepidemiology 32, 150-163 (2009)

26. Islam, Z. et al. High incidence of Guillain-Barré syndrome in children, Bangladesh. Emerg. Infect. Dis. 17, 1317-1318 (2011)

27. Hughes, R. A. \& Cornblath, D. R. Guillain-Barré syndrome. Lancet 366, 1653-1666 (2005).
28. Van Koningsveld, R. et al. Gastroenteritis-associated Guillain-Barré syndrome on the Caribbean island Curacao. Neurology 56, 1467-1472 (2001).

29. Webb, A. J., Brain, S. A., Wood, R., Rinaldi, S. \& Turner, M. R. Seasonal variation in Guillain-Barré syndrome: a systematic review, meta-analysis and Oxfordshire cohort study. J. Neurol. Neurosurg. Psychiatry 86, 1196-1201 (2015).

30. Wakerley, B. R. \& Yuki, N. Infectious and noninfectious triggers in Guillain-Barré syndrome. Expert Rev. Clin. Immunol. 9, 627-639 (2013).

31. Vellozzi, C., Iqbal, S. \& Broder, K. Guillain-Barré syndrome, influenza, and influenza vaccination: the epidemiologic evidence. Clin. Infect. Dis. 58, 1149-1155 (2014).

32. Ho, T. et al. Guillain-Barré syndrome in northern China: relationship to Campylobacter jejuni infection and antiglycolipid antibodies. Brain 118, 597-605 (1995).

33. Platts-Mills, J. A. et al. Pathogen-specific burdens of community diarrhoea in developing countries: a multisite birth cohort study (MAL-ED). Lancet Glob. Health 3, e564-e575 (2015).

34. Larrosa-Haro, A., Macias-Rosales, R., Sánchez-Ramírez, C. A., Cortés-López, M. C. $\&$ Aguilar-Benavides, S. Seasonal variation of enteropathogens in infants and preschoolers with acute diarrhea in western Mexico. J. Pediatr. Gastroenterol. Nutr. 51, 534-536 (2010).

35. Bahemuka, M. Guillain-Barré syndrome in Kenya: a clinical review of 54 patients. J. Neurol. 235, 418-421 (1988)

36. Charra, B., Hachimi, A., Benslama, A. \& Motaouakkil, S. Intravenous immunoglobulin vs. plasma exchange in treatment of mechanically ventilated adults with Guillain-Barrê syndrome. Pan Afr. Med. J. 18, 35 (2014).

37. Cheng, B. C. et al. Guillain-Barré syndrome in southern Taiwan: clinical features, prognostic factors and therapeutic outcomes. Eur. J. Neurol. 10, 655-662 (2003).

38. Howlett, W., Vedeler, C., Nyland, H. \& Aarli, J. GuillainBarré syndrome in northern Tanzania: a comparison of epidemiological and clinical findings with western Norway. Acta Neurol. Scand. 93, 44-49 (1996). 
39. Liou, L.-S. et al. Epidemiology and prognostic factors of inpatient mortality of Guillain-Barrê syndrome: a nationwide population study over 14 years in Asian country. J. Neurol. Sci. 369, 159-164 (2016).

40. Melaku, Z., Zenebe, G. \& Bekele, A. Guillain-Barré syndrome in Ethiopian patients. Ethiopian Med. J. 43 21-26 (2005)

41. Netto, A. B., Taly, A. B., Kulkarni, G. B., Rao, U. G. \& Rao, S. Mortality in mechanically ventilated patients of Guillain-Barré syndrome. Ann. Indian Acad. Neurol. 14, 262-266 (2011).

42. Siddiqui, S. H., Siddiqui, T. H., Babar, M. U., Khoja, A. $\&$ Khan, S. Outcomes of patients with GuillainBarré syndrome - experience from a tertiary care hospital of a developing Asian country and review of regional literature. J. Clin. Neurosci. 62, 195-198 (2019).

43. Wierzba, T. F. et al. Campylobacter infection as a trigger for Guillain-Barrē syndrome in Egypt. PLOS ONE 3, e3674 (2008).

44. Dourado, M., Felix, R., da Silva, W., Queiroz, J. \& Jeronimo, S. Clinical characteristics of Guillain-Barré syndrome in a tropical country: a Brazilian experience. Acta Neurol. Scand. 125, 47-53 (2012).

45. Meena, A., Khadilkar, S. \& Murthy, J. Treatment guidelines for Guillain-Barré syndrome. Ann. Indian Acad. Neurol. 14, S73-S81 (2011)

46. Kaakoush, N. O., Castaño-Rodrīguez, N. Mitchell, H. M. \& Man, S. M. Global epidemiology of Campylobacter infection. Clin. Microbiol. Rev. 28 687-720 (2015).

47. van Koningsveld, R. et al. A clinical prognostic scoring system for Guillain-Barré syndrome. Lancet Neurol. 6 589-594 (2007)

48. Bae, J. S. et al. Guillain-Barré syndrome in Asia. J. Neurol. Neurosurg. Psychiatry 85, 907-913 (2014).

49. Mitsui, Y. et al. A multicentre prospective study of Guillain-Barré syndrome in Japan: a focus on the incidence of subtypes. J. Neurol. Neurosurg. Psychiatry 86, 110-114 (2015)

50. Ng, Y., Lo, Y. \& Lim, P. Characteristics and acute rehabilitation of Guillain-Barré syndrome in Singapore. Ann. Acad. Med. Singap. 33, 314-319 (2004).

51. Koga, M., Yuki, N., Tai, T. \& Hirata, K. Miller Fisher syndrome and Haemophilus influenzae infection. Neurology 57, 686-691 (2001).

52. Zhang, G. et al. Subtypes and prognosis of GuillainBarré syndrome in southwest China. PLOS ONE 10, e0133520 (2015).

53. Principi, N. \& Esposito, S. Vaccine-preventable diseases, vaccines and Guillain-Barré syndrome. Vaccine 37, 5544-5550 (2019).

54. Rudant, J. et al. Surgery and risk of Guillain-Barré syndrome: a French nationwide epidemiologic study. Neurology 91, e1220-e1227 (2018)

55. Hiew, F. L. \& Rajabally, Y. A. Malignancy in GuillainBarré syndrome: a twelve-year single-center study. J. Neurol. Sci. 375, 275-278 (2017).

56. Shafqat, S., Khealani, B., Awan, F. \& Abedin, S Guillain-Barre syndrome in Pakistan: similarity of demyelinating and axonal variants. Eur. J. Neurol. 13, 662-665 (2006)

57. Sudulagunta, S. R. et al. Guillain-Barré syndrome: clinical profile and management. Ger. Med. Sci. 13 Doc16 (2015).

58. Poropatich, K. O., Walker, C. L. F. \& Black, R. E. Quantifying the association between Campylobacter infection and Guillain-Barré syndrome: a systematic review. J. Health Popul. Nutr. 28, 545-552 (2010).

59. Hao, Y. et al. Antecedent infections in Guillain-Barré syndrome: a single-center, prospective study. Ann. Clin. Transl. Neurol. 6, 2510-2517 (2019).

60. Willison, H. J. \& O'Hanlon, G. M. The immunopathogenesis of Miller Fisher syndrome. J. Neuroimmunol. 100, 3-12 (1999).

61. Takahashi, M., Koga, M., Yokoyama, K. \& Yuki, N Epidemiology of Campylobacter jejuni isolated from patients with Guillain-Barré and Miller Fisher syndromes in Japan. J. Clin. Microbiol. 43, 335-339 (2005).

62. Baker, M. G. et al. Declining Guillain-Barré syndrome after campylobacteriosis control, New Zealand, 1988-2010. Emerg. Infect. Dis. 18, 226-233 (2012).

63. Styczynski, A. R. et al. Increased rates of GuillainBarré syndrome associated with Zika virus outbreak in the Salvador metropolitan area, Brazil. PLoS Negl. Trop. Dis. 11, e0005869 (2017).
64. Kanjalkar, M., Karnad, D., Narayana, R. \& Shah, P. Guillain-Barré syndrome following malaria. J Infect. 38, 48-50 (1999).

65. Sithinamsuwan, P., Sinsawaiwong, S. \& Limapichart, K. Guillain-Barrê's syndrome associated with Plasmodium falciparum malaria: role of plasma exchange. J. Med. Assoc. Thai. 84, 1212-1216 (2001).

66. Wijesundere, A. Guillain-Barré syndrome in Plasmodium falciparum malaria. Postgrad. Med. J. 68, 376-377 (1992)

67. Thornton, C. A., Latif, A. S. \& Emmanuel, J. C. Guillain-Barre syndrome associated with human immunodeficiency virus infection in Zimbabwe. Neurology 41, 812 (1991).

68. Gupta, P., Jain, V., Chatterjee, S. \& Agarwal, A. Acute inflammatory motor axonopathy associated with dengue fever. J. Indian Acad. Clin. Med. 10, 58-59 (2009).

69. Ralapanawa, D. M. P. U. K., Kularatne, S. A. M. \& Jayalath, W. A. T. A. Guillain-Barré syndrome following dengue fever and literature review. BMC Res. Notes 8, 729 (2015)

70. Santos, N. Q., Azoubel, A. C. B., Lopes, A. A., Costa, C. $\&$ Bacellar, A. Guillain-Barré syndrome in the course of dengue: case report. Arq. Neuropsiquiatr. 62, 144-146 (2004)

71. Sedaghat, Z. \& Karimi, N. Guillain-Barré syndrome associated with COVID-19 infection: a case report. J. Clin. Neurosci. 76, 233-235 (2020).

72. Toscano, G. et al. Guillain-Barrê syndrome associated with SARS-CoV-2. N. Engl. J. Med. 382, 2574-2576 (2020).

73. Zhao, H., Shen, D., Zhou, H., Liu, J. \& Chen, S. Guillain-Barre syndrome associated with SARS-CoV-2 infection: causality or coincidence? Lancet Neurol. 19, 383-384 (2020)

74. Camdessanche, J.-P. et al. COVID-19 may induce Guillain-Barrê syndrome. Rev. Neurol. 176, 516-518 (2020).

75. Nanda, S. et al. Covid-19 associated Guillain-Barré syndrome: contrasting tale of four patients from tertiary care centre in India. Am. J. Emerg. Med. 39 125-128 (2020)

76. Sidig, A., Abbasher, K., Abbasher, H., Abbasher, M. \& Hussien, A. COVID-19 and Guillain-Barré syndrome case report. J. Neurol. Neurobiol. 7, https://doi.org 10.16966/2379-7150.169 (2020)

77. El Otmani, H. et al. Covid-19 and Guillain-Barré syndrome: more than a coincidence! Rev. Neurol. 176 518-519 (2020)

78. Keddie, S. et al. Epidemiological and cohort study finds no association between COVID-19 and GuillainBarré syndrome. Brain https://doi.org/10.1093/brain/ awaa433 (2020)

79. Muñoz, L. S., Parra, B., Pardo, C. A. \& Neuroviruses Emerging in the Americas Study. Neurological implications of Zika virus infection in adults. J. Infect. Dis. 216 (Suppl. 10), S897-S905 (2017).

80. Uncini, A., Vallat, J.-M. \& Jacobs, B. C. Guillain-Barre syndrome in SARS-CoV-2 infection: an instant systematic review of the first six months of pandemic. J. Neurol. Neurosurg. Psychiatry 91, 1105-1110 (2020).

81. Abu-Rumeileh, S., Abdelhak, A., Foschi, M., Tumani, H. ¿ Otto, M. Guillain-Barré syndrome spectrum associated with COVID-19: an up-to-date systematic review of 73 cases. J. Neurol. https://doi.org/10.1007/ s00415-020-10124-x (2020).

82. Blum, S. \& McCombe, P. A. Genetics of Guillain-Barre syndrome (GBS) and chronic inflammatory demyelinating polyradiculoneuropathy (CIDP): current knowledge and future directions. J. Peripher Nerv. Syst. 19, 88-103 (2014)

83. Caporale, C. M. et al. Susceptibility to Guillain-Barré syndrome is associated to polymorphisms of $C D$ genes. J. Neuroimmunol. 177, 112-118 (2006).

84. Islam, Z. et al. FAS promoter polymorphisms and serum sFas level are associated with increased risk of nerve damage in Bangladeshi patients with GuillainBarrê syndrome. PLOS ONE 13, e0192703 (2018).

85. Harbige, L. S. Nutrition and immunity with emphasis on infection and autoimmune disease. Nutr. Health 10, 285-312 (1996).

86. Díaz-Soto, S., Chavez, K., Chaca, A., Alanya, J. ¿ Tirado-Hurtado, I. Outbreak of Guillain-Barré syndrome in Peru. eNeurologicalSci 14, 89-90 (2019).

87. Dirlikov, E. et al. Guillain-Barrê syndrome and healthcare needs during Zika virus transmission, Puerto Rico, 2016. Emerg. Infect. Dis. 23, 134-136 (2017).
88. Mier-y-Teran-Romero, L., Delorey, M. J., Sejvar, J. J. \& Johansson, M. A. Guillain-Barrē syndrome risk among individuals infected with Zika virus: a multi-country assessment. BMC Med. 16, 67 (2018).

89. Jones, K. E. et al. Global trends in emerging infectious diseases. Nature 451, 990-993 (2008)

90. Ferreira, M. L. B. et al. Neurological disease in adults with Zika and chikungunya virus infection in Northeast Brazil: a prospective observational study. Lancet Neurol. 19, 826-839 (2020).

91. Simon, O. et al. Early Guillain-Barré syndrome associated with acute dengue fever. J. Clin. Virol. 77. 29-31 (2016)

92. Lowe, R. et al. The Zika virus epidemic in Brazil: from discovery to future implications. Int. J. Environ. Res. Publ. Health 15, 96 (2018).

93. Zhang, Y. et al. Epidemiological survey of the incidence of Guillain-Barré syndrome in Harbin from 1997 to 1999. Chin. J. Clin. Rehab. 34, 7812-7815 (2004).

94. Leonhard, S. E., Cornblath, D. R., Endtz, H. P., Sejvar, J. J. \& Jacobs, B. C. Guillain-Barré syndrome in times of pandemics. J. Neurol. Neurosurg. Psychiatry 91, 1027-1029 (2020)

95. Asbury, A. K. ¿ Cornblath, D. R. Assessment of current diagnostic criteria for Guillain-Barre syndrome. Ann. Neurol 27 S21-S24 (1990).

96. Islam, M. B. et al. Guillain-Barré syndrome in Bangladesh: validation of Brighton criteria J. Peripher. Nerv. Syst. 21, 345-351 (2016).

97. Lehmann, H. C., Hughes, R. A., Kieseier, B. C. \& Hartung, H. P. Recent developments and future directions in Guillain-Barrê syndrome. J. Peripher. Nerv. Syst. 17, 57-70 (2012)

98. Mateen, F. J. et al. Guillain-Barré syndrome in India: population-based validation of the Brighton criteria. Vaccine 29, 9697-9701 (2011).

99. Zeng, Y. et al. Clinical features and the validation of the Brighton criteria in Guillain-Barre syndrome retrospective analysis of 72 hospitalized patients in three years. Eur. Neurol. 81, 231-238 (2019).

100. Yuki, N. et al. Guillain-Barré syndrome associated with normal or exaggerated tendon reflexes. J. Neurol. 259, 1181-1190 (2012).

101. Jacobs, B. et al. The spectrum of antecedent infections in Guillain-Barré syndrome: a case-control study. Neurology 51, 1110-1115 (1998)

102. Roodbol, J. et al. Recognizing Guillain-Barré syndrome in preschool children. Neurology 76 807-810 (2011)

103. Korinthenberg, R., Schessl, J. \& Kirschner, J. Clinical presentation and course of childhood Guillain-Barré syndrome: a prospective multicentre study. Neuropediatrics 38, 10-17 (2007).

104. Khan, F., Ng, L., Amatya, B., Brand, C. $\delta$ Turner-Stokes, L. Multidisciplinary care for GuillainBarré syndrome. Eur. J. Phys. Rehabil. Med. 47. 607-612 (2011).

105. Verboon, C., van Doorn, P. A. \& Jacobs, B. C. Treatment dilemmas in Guillain-Barré syndrome. J. Neurol. Neurosurg. Psychiatry 88, 346-352 (2017).

106. Chaudhuri, J. R. et al. Clinical outcome of GuillainBarré syndrome with various treatment methods and cost effectiveness: a study from tertiary care center in South India: Yashoda GBS registry. Neurol. Asia 19 263-270 (2014).

107. Willison, H. J., Jacobs, B. C. \& van Doorn, P. A Guillain-Barré syndrome: surveillance and cost of treatment strategies - authors' reply. Lancet 389 253-254 (2017)

108. Kishore, C. K et al. Management of Guillain-Barré syndrome with plasmapheresis or immunoglobulin: our experience from a tertiary care institute in South India. Ren. Fail. 36, 732-736 (2014)

109. Islam, Z. et al. Risk factors for respiratory failure in Guillain-Barré syndrome in Bangladesh: a prospective study. Ann. Clin. Transl. Neurol. 6, 324-332 (2019).

110. Iyer, R. R., Shah, P. H., Roy, S. S. K. \& Suri, S. K. K. Reducing the economic burden in management of Guillain-Barré syndrome using modified plasmapheresis. Asian J. Transfus. Sci. 10, 118-121 (2016).

111. Islam, Z. et al. Inhibition of C1q, initiator of the classical complement cascade, by ANX005 for the treatment of Guillain-Barré syndrome: results from a phase 1b study [abstract]. Neurology 94 (Suppl. 15), 763 (2020).

112. Lucchini, S. et al. in Economics of AIDS and Access to HIV-AIDS Care in Developing Countries: Issues and Challenges (eds Moatti, J. P. et al.) 169-211 (ANRS, 2003) 
113. Kalita, J., Ranjan, A. \& Misra, U. Outcome of GuillainBarré syndrome patients with respiratory paralysis. OJM 109, 319-323 (2016).

114. Leonhard, S. E. et al. Diagnosis and management of Guillain-Barré syndrome in ten steps. Nat. Rev. Neurol. 15, 671-683 (2019).

115. Wu, X. et al. Predictors for mechanical ventilation and short-term prognosis in patients with Guillain-Barré syndrome. Crit. Care 19, 407 (2015).

116. Walgaard, C. et al. Prediction of respiratory insufficiency in Guillain-Barrê syndrome. Ann. Neurol. 67, 781-787 (2010).

117. Adhikary, T. S. $\&$ Mollah, S. ICU facilities scanty at government hospitals of Bangladesh. The Daily Star (Bangladesh) (6 Feb 2021).

118. Gonzălez-Suărez, I., Sanz-Gallego, I., de Rivera, F. J. R. ¿ Arpa, J. Guillain-Barrē syndrome: natural history and prognostic factors: a retrospective review of 106 cases. BMC Neurol. 13, 95 (2013).

119. Halawa, E. F., Ahmed, D. \& Nada, M. A. GuillainBarré syndrome as a prominent cause of childhood acute flaccid paralysis in post polio eradication era in Egypt. Eur. J. Paediatr. Neurol. 15, 241-246 (2011).

120. Bright, T., Wallace, S. \& Kuper, H. A systematic review of access to rehabilitation for people with disabilities in low- and middle-income countries. Int. J. Environ. Res. Publ. Health 15, 2165 (2018)

121. Rajabally, Y. A. \& Uncini, A. Outcome and its predictors in Guillain-Barré syndrome. J. Neurol. Neurosurg. Psychiatry 83, 711-718 (2012).

122. Hiraga, A. et al. Recovery patterns and long term prognosis for axonal Guillain-Barré syndrome. J. Neurol. Neurosurg. Psychiatry 76, 719-722 (2005).

123. Merkies, I. S. \& Kieseier, B. C. Fatigue, pain, anxiety and depression in Guillain-Barré syndrome and chronic inflammatory demyelinating polyradiculoneuropathy. Eur. Neurol. 75, 199-206 (2016).

124. Khan, F., Pallant, J., Ng, L. \& Bhasker, A. Factors associated with long-term functional outcomes and psychological sequelae in Guillain-Barrê syndrome. J. Neurol. 257, 2024-2031 (2010).
125. Walgaard, C. et al. Early recognition of poor prognosis in Guillain-Barré syndrome. Neurology 76, 968-975 (2011).

126. Dourado Júnior, M. E. et al. EGOS has a reduced capacity to predicts GBS prognosis in Northeast Brazil. Acta Neurol. Scand. 138, 459-462 (2018).

127. Yamagishi, Y. et al. Markers for Guillain-Barrê syndrome with poor prognosis: a multi-center study. J. Peripher. Nerv. Syst. 22, 433-439 (2017).

128. Tan, C. Y., Razali, S. N., Goh, K. J. \& Shahrizaila, N. The utility of Guillain-Barre syndrome prognostic models in Malaysian patients. J. Peripher. Nerv. Syst. 24, 168-173 (2019)

129. Graham, R. C. \& Hughes, R. A modified peripheral neuropathy scale: the overall neuropathy limitations scale. J. Neurol. Neurosurg. Psychiatry 77, 973-976 (2006).

130. Lunn, M. P. \& Van den Bergh, P. Y. Outcome measures in neuromuscular disease: is the world still flat? J. Peripher. Nerv. Syst. 20, 255-259 (2015).

131. Van Nes, S. I. et al. Improving fatigue assessment in immune-mediated neuropathies: the modified RaschBuilt fatigue severity scale. J. Peripher. Nerv. Syst. 14, 268-278 (2009)

132. Bhagat, S. K., Sidhant, S., Bhatta, M., Ghimire, A. $\&$ Shah, B. Clinical profile, functional outcome, and mortality of Guillain-Barré syndrome: a five-year tertiary care experience from Nepal. Neurol. Res. Int. 2019, 3867946 (2019)

133. Park, Y.-S., Lee, K.-J., Kim, S. W., Kim, K. M. \& Suh, B. C. Clinical features of post-vaccination Guillain-Barré syndrome (GBS) in Korea. J. Korean Med. Sci. 32 1154-1159 (2017)

Acknowledgements

We thank H.U.R Javid, M.L. Brito, FA A Gondim, M.-E. Dourado, Y. Wang, S. Elaidy, T.C. Yin, D.R. Ohnmar, M.A. UI Haq, A.P. Ramos, T. Thomas, K. Bateman, A. Keshavaraj, M. Dekker, W. Howlett, Hoa Nguyen, Hieu Nguyen, S. Khan K. Prasad, U. Thirugnanam, M. Hakim, B. Alam and G. Mondol for providing comments and completing questionnaires on the differential diagnosis of GBS in their region. The research work of S.E.L. and B.C.J. is supported by ZikaPLAN a global research consortium funded by the European Union under the Horizon 2020 programme (grant number 734584). Z.I. received restricted grant (number 1K43TW011447-01) support from Fogarty International Center, the National Institute of Neurological Disorders and Stroke (NINDS), National Institutes of Health $(\mathrm{NIH})$ and Annexon Biosciences for activities unrelated to the subject matter of this Review. Q.D.M. received consulting honoraria from Annexon Biosciences for activities unrelated to the subject matter of this Review. B.C.J. received unrestricted support for research from Annexon Biosciences, CSL-Behring, EU Horizon 2020, Griffols, Hansa Biopharma and Prinses Beatrix Spierfonds, for activities unrelated to the subject matter of this Review.

\section{Author contributions}

All authors contributed substantially to the initial discussions of the article content and to review or editing of the manuscript before submission. N.P. conducted the literature search, and N.P. and Z.I. researched data for the article. N.P. and Z.I. wrote the first draft of the manuscript and S.E.L.,

D.M., H.P.E. and B.C.J. contributed to subsequent versions.

Competing interests

The authors declare no competing interests.

Peer review information

Nature Reviews Neurology thanks T. Umapathi and the other, anonymous, reviewers for their contribution to the peer review of this work.

\section{Publisher's note}

Springer Nature remains neutral with regard to jurisdictional claims in published maps and institutional affiliations.

\section{Supplementary information}

The online version contains supplementary material available at https://doi.org/10.1038/s41582-021-00467-y.

\section{RELATED LINKS}

GBS|CIDP Foundation International: https://www.gbs-cidp.org/

(c) Springer Nature Limited 2021 